Revista de Matemática: Teoría y Aplicaciones 2016 23(1) : 277-289

CIMPA - UCR ISSN: 1409-2433 (PRINT), 2215-3373 (ONLINE)

\title{
ORDERING CATACONDENSED HEXAGONAL SYSTEMS WITH RESPECT TO VDB TOPOLOGICAL INDICES
}

\section{ORDENACIÓN DE LOS SISTEMAS HEXAGONALES CATACONDENSADOS CON RESPECTO A LOS ÍNDICES TOPOLÓGICOS VDB}

\author{
JUAN RADA*
}

Received: 24 Jul 2014; Revised: 14 Oct 2015;

Accepted: 19 Oct 2015

*Instituto de Matemáticas, Universidad de Antioquia, Medellín, Colombia. E-Mail: pablo.rada@udea.edu.co 


\begin{abstract}
In this paper we give a complete description of the ordering relations in the set of catacondensed hexagonal systems, with respect to a vertexdegree-based topological index. As a consequence, extremal values of vertex-degree-based topological indices in special subsets of the set of catacondensed hexagonal systems are computed.
\end{abstract}

Keywords: VDB topological indices; catacondensed hexagonal systems; ordering; extremal values.

\title{
Resumen
}

En este artículo presentamos una descripción completa de la relación de orden en el conjunto de sistemas hexagonales catacondensados, con respecto a un índice topológico basado en los grados de los vértices. Como consecuencia, se determinan los valores extremos de un índice topológico basados en los grados de los vértices en subconjuntos especiales del conjunto de sistemas hexagonales catacondensados.

Palabras clave: índices topológicos VDB; sistemas hexagonales catacondensados; orden; valores extremos.

Mathematics Subject Classification: 05C12, $05 \mathrm{C} 05$.

\section{Introduction}

A vertex-degree-based topological index (VDB for short) is denoted by $T I$ and defined from a set of real numbers $\left\{\varphi_{i j}\right\}$, for $1 \leq i \leq j \leq n-1$, as

$$
T I(G)=\sum_{1 \leq i \leq j \leq n-1} m_{i j} \varphi_{i j}
$$

where $G$ is a graph (i.e. undirected graph) with $n$ vertices and $m_{i j}=m_{i j}(G)$ the number of $i j$-edges, i.e. the number of edges in $G$ with end vertices of degree $i$ and $j$ ([11], [10], [18]). When $\varphi_{i j}=\frac{1}{\sqrt{i j}}$ we obtain the Randić index ([20]), one of the most widely used in applications to physical and chemical properties ([3],[12],[13],[21]). For a recent survey on the mathematical properties of the Randić index we refer to $([14],[15])$. Due to the success of the Randić index many other topological indices appeared in the mathematical-chemistry literature, which are particular cases of the formula given in (1), as we can see in Table 1.

We will study VDB topological indices over hexagonal systems, natural graph representations of benzenoid hydrocarbons which are of great importance 
in chemistry. For further results on VDB topological indices over hexagonal systems we refer to $([1],[2],[17],[19])$. Recall that a hexagonal system is a finite connected plane graph without cut vertices, in which all interior regions are mutually congruent regular hexagons. The inner dual of an hexagonal system $G$, denoted by $I D(G)$, is the graph whose vertices are the hexagons of $G$ and two vertices are adjacent in $I D(G)$, if the correspondent hexagons are adjacent in $G$. A catacondensed hexagonal system (resp. hexagonal chain) is an hexagonal system whose inner dual graph is a tree (resp. a path). The hexagons in a catacondensed system are classified as linear $\left(L_{1}\right.$ and $\left.L_{2}\right)$ or angular $\left(A_{2}\right.$ and $\left.A_{3}\right)$, according to the number and position of edges shared with the adjacent hexagons (See Figure 1). More details on this class of graphs can be found in [9].

Table 1: Some well-known VDB topological indices.

\begin{tabular}{l|cc}
\hline \hline Index & Notation & $\varphi_{i j}$ \\
\hline First Zagreb [7] & $\mathcal{F Z}$ & $i+j$ \\
Second Zagreb [7] & $\mathcal{S Z}$ & $i j$ \\
Randić [20] & $\chi$ & $\frac{1}{\sqrt{i j}}$ \\
Harmonic [24] & $\mathcal{H}$ & $\frac{2}{i+j}$ \\
Geometric-Arithmetic [22] & $\mathcal{G A}$ & $\frac{2 \sqrt{i j}}{i+j}$ \\
Sum-Connectivity [23] & $\mathcal{S C}$ & $\frac{1}{\sqrt{i+j}}$ \\
Atom-Bond-Connectivity [4] & $\mathcal{A B C}$ & $\sqrt{\frac{i+j-2}{i j}}$ \\
Augmented Zagreb [5] & $\mathcal{A Z}$ & $\left(\frac{i j}{i+j-2}\right)^{3}$ \\
\hline \hline
\end{tabular}

In this paper we give a complete description of the order relation in the set of catacondensed hexagonal systems with respect to a VDB topological index TI. As a consequence, extremal values of $T I$ in special subsets of the set of of catacondensed hexagonal systems with a fixed number of hexagons are computed. 


\section{Ordering catacondensed hexagonal systems induced by VDB topological indices}

We denote by $\mathcal{C H}_{h}$ the set of catacondensed hexagonal systems with $h$ hexagons. Since every $W \in \mathcal{C H}_{h}$ has only vertices of degree 2 and 3 , a topological index $T I$ defined from the numbers $\left\{\varphi_{i j}\right\}$ as in (1) can be expressed as

$$
T I(W)=m_{22} \varphi_{22}+m_{23} \varphi_{23}+m_{33} \varphi_{33} .
$$

From now on we denote by $a_{2}(W), a_{3}(W), l_{1}(W)$ and $l_{2}(W)$ the number of $A_{2}, A_{3}, L_{1}$ and $L_{2}$ hexagons $W$ has, respectively. If it is clear from the context we just write $a_{2}, a_{3}, l_{1}$ and $l_{2}$.

Lemma 2.1 Let $W \in \mathcal{C H}_{h}$. Then

$$
\begin{aligned}
& m_{22}=a_{2}+3 a_{3}+6 \\
& m_{23}=4(h-1)-2 a_{2}-6 a_{3} \\
& m_{33}=h-1+3 a_{3}+a_{2} .
\end{aligned}
$$

Proof. The following relations are well-known [8]

$$
\begin{aligned}
& m_{22}=a_{2}+3 a_{3}+6 \\
& m_{23}=4 l_{1}+4 l_{2}+2 a_{2}-2 a_{3}-4 \\
& m_{33}=l_{1}+l_{2}+2 a_{2}+4 a_{3}-1 .
\end{aligned}
$$

Since

$$
\begin{gathered}
l_{1}=a_{3}+2 \\
h=l_{1}+l_{2}+a_{2}+a_{3}
\end{gathered}
$$

we deduce

$$
\begin{aligned}
l_{2} & =h-l_{1}-a_{2}-a_{3} \\
& =h-\left(a_{3}+2\right)-a_{2}-a_{3} \\
& =h-2 a_{3}-a_{2}-2 .
\end{aligned}
$$

Now substituting the expressions

$$
\begin{aligned}
& l_{1}=a_{3}+2 \\
& l_{2}=h-2 a_{3}-a_{2}-2
\end{aligned}
$$

in (3) gives the result.

The following result was shown in [17] using linearizing and unbranching operations over catacondensed hexagonal systems. Now we simplify the proof based on the combinatorial arguments given in Lemma 2.1. 
Proposition 2.2 Let TI be a VDB topological index induced by the numbers $\left\{\varphi_{i j}\right\}$ and $W \in \mathcal{C H}_{h}$. Then

$$
\begin{aligned}
T I(W)=\left(\varphi_{22}-2 \varphi_{23}+\varphi_{33}\right)\left(a_{2}\right. & \left.+3 a_{3}\right)+\left(4 \varphi_{23}+\varphi_{33}\right) h \\
& +\left(6 \varphi_{22}-4 \varphi_{23}-\varphi_{33}\right) .
\end{aligned}
$$

Proof. From the expression given in (2) and Lemma2.1,

$$
\begin{aligned}
T I(W)= & m_{22} \varphi_{22}+m_{23} \varphi_{23}+m_{33} \varphi_{33} \\
= & \left(a_{2}+3 a_{3}+6\right) \varphi_{22}+\left(4(h-1)-2 a_{2}-6 a_{3}\right) \varphi_{23} \\
& +\left(h-1+3 a_{3}+a_{2}\right) \varphi_{33} \\
= & \left(\varphi_{22}-2 \varphi_{23}+\varphi_{33}\right)\left(a_{2}+3 a_{3}\right)+\left(4 \varphi_{23}+\varphi_{33}\right) h \\
& +\left(6 \varphi_{22}-4 \varphi_{23}-\varphi_{33}\right) .
\end{aligned}
$$

Consider the subset of $\mathcal{C} \mathcal{H}_{h}$ denoted by $\mathcal{C} \mathcal{H}_{h, p}$ and defined by

$$
\mathcal{C H}_{h, p}=\left\{W \in \mathcal{C H}_{h}: a_{3}(W)=p\right\}
$$

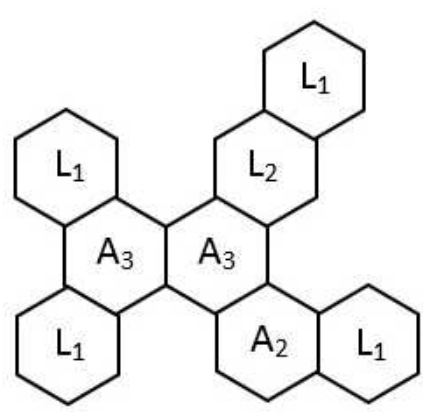

W

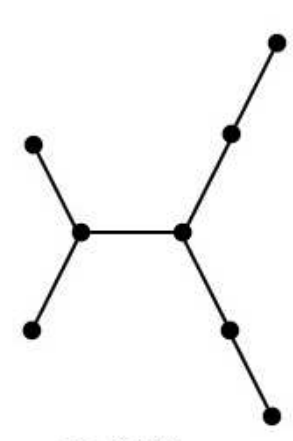

$\mathrm{ID}(\mathrm{W})$

Figure 1: Catacondensed hexagonal system and its inner dual graph.

Example 2.3 The hexagonal system $W$ in Figure 1 belongs to $\mathcal{C H}_{8,2}$. Note that $\mathrm{CH}_{h, 0}$ consists of all hexagonal chains with h hexagons. In particular, the linear hexagonal chain $L_{h}$ and the zig-zag chain $Z_{h}$ belong to $\mathcal{C H} \mathcal{H}_{h, 0}$ (see Figure 2). The hexagonal system $E_{h}$ belongs to $\mathcal{C H}_{h,\left\lfloor\frac{1}{2}(h-2)\right\rfloor}$ (see Figure 3). 


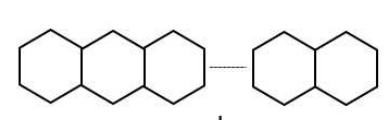

$L_{h}$

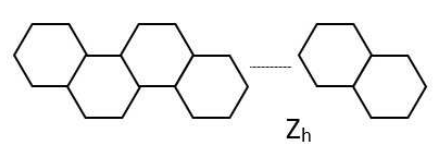

$Z_{h}$

Figure 2: The linear chain $L_{h}$ and the zig-zag chain $Z_{h}$.

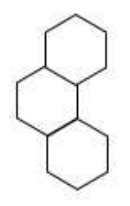

$E_{3}$

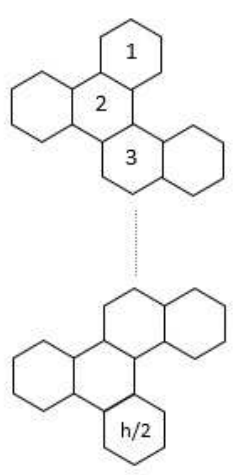

Eh (h even, $h>4$ )

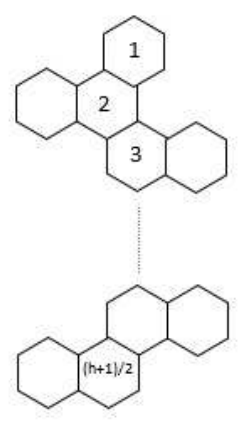

Eh ( $h$ odd, $h>3$ )

Figure 3: The hexagonal system $E_{h}$.

Lemma 2.4 Let $h \geq 3$ and $W \in \mathcal{C H}_{h}$. Then

$$
0 \leq a_{3}(W) \leq\left\lfloor\frac{1}{2}(h-2)\right\rfloor .
$$

Moreover, equality on the left is attained in hexagonal chains and the equality on the right is attained in $E_{h}$ (see Figure 3).

Proof. From the relations given in (4) we deduce

$$
a_{3}=\frac{1}{2}\left(h-\left(a_{2}+l_{2}+2\right)\right) .
$$

If $h$ is even then it follows from (5)

$$
a_{3} \leq \frac{1}{2}(h-2)
$$

since $a_{2}+l_{2} \geq 0$. If $h$ is odd then again by (5) $a_{2}+l_{2} \geq 1$, since $a_{2}+l_{2}=0$ implies that $a_{3}$ is not an integer, a contradiction. Hence

$$
a_{3} \leq \frac{1}{2}(h-3) .
$$


The equality on the left is clear for hexagonal chains. On the other hand, note that $a_{2}\left(E_{h}\right)=0=l_{2}\left(E_{h}\right)$ if $h$ is even, $a_{2}\left(E_{h}\right)=1$ and $l_{2}\left(E_{h}\right)=0$ if $h$ is odd. It follows from (5) that

$$
a_{3}\left(E_{h}\right)= \begin{cases}\frac{1}{2}(h-2) & \text { if } h \text { is even } \\ \frac{1}{2}(h-3) & \text { if } h \text { is odd. }\end{cases}
$$

Lemma 2.5 Let $h \geq 3$ and $0 \leq p \leq\left\lfloor\frac{1}{2}(h-2)\right\rfloor$. If $W \in \mathcal{C H}_{h, p}$ then

$$
0 \leq a_{2}(W) \leq h-2(p+1)
$$

Proof. From relations (4) and the fact that $l_{2} \geq 0$ we deduce

$$
a_{2}=h-2 p-l_{2}-2 \leq h-2(p+1) .
$$

Let us denote by $\mathcal{C H}_{h, p, q}$ the subset of $\mathcal{C H}_{h, p}$ defined as

$$
\mathcal{C H}_{h, p, q}=\left\{W \in \mathcal{C H}_{h, p}: a_{2}(W)=q\right\}
$$

Clearly if $T I$ is a VDB topological index induced by the numbers $\left\{\varphi_{i j}\right\}$ then by Proposition 2.2, TI is constant over $\mathcal{C H}_{h, p, q}$. We will use the notation

$$
T I\left(\mathcal{C H}_{h, p, q}\right) \leq T I\left(\mathcal{C H}_{h, p^{\prime}, q^{\prime}}\right)
$$

to indicate that $T I(U) \leq T I(V)$ for all $U \in \mathcal{C} \mathcal{H}_{h, p, q}$ and $V \in \mathcal{C} \mathcal{H}_{h, p^{\prime}, q^{\prime}}$. The following result gives a complete description of the order relations in the set of catacondensed hexagonal systems with respect to a VDB topological index.

Theorem 2.6 Let TI be a VDB topological index induced by the numbers $\left\{\varphi_{i j}\right\}$. Let $h \geq 3$ and $0 \leq p \leq\left\lfloor\frac{1}{2}(h-2)\right\rfloor$. Assume that $\varphi_{22}-2 \varphi_{23}+\varphi_{33}>0$. Then

1. $T I\left(\mathcal{C H}_{h, p, i}\right)<T I\left(\mathcal{C H}_{h, p, i+1}\right)$ for all $i=0, \ldots, h-2 p-3$.

2. $T I\left(\mathcal{C H}_{h, p, q}\right)<T I\left(\mathcal{C H}_{h, p+1, q^{\prime}}\right)$ if and only if $q^{\prime}>q-3$.

3. $T I\left(\mathcal{C H}_{h, p, q}\right)=T I\left(\mathcal{C H}_{h, p+1, q^{\prime}}\right)$ if and only if $q^{\prime}=q-3$. 
Proof. 1. Let $U \in \mathcal{C H}_{h, p, i}$ and $V \in \mathcal{C} \mathcal{H}_{h, p, i+1}$. Then by Proposition 2.2

$$
\begin{aligned}
T I(U)= & \left(\varphi_{22}-2 \varphi_{23}+\varphi_{33}\right)(i+3 p)+\left(4 \varphi_{23}+\varphi_{33}\right) h \\
& +\left(6 \varphi_{22}-4 \varphi_{23}-\varphi_{33}\right) \\
T I(V)= & \left(\varphi_{22}-2 \varphi_{23}+\varphi_{33}\right)(i+1+3 p)+\left(4 \varphi_{23}+\varphi_{33}\right) h \\
& +\left(6 \varphi_{22}-4 \varphi_{23}-\varphi_{33}\right) .
\end{aligned}
$$

Consequently

$$
T I(U)-T I(V)=\left(\varphi_{22}-2 \varphi_{23}+\varphi_{33}\right)(-1)<0 .
$$

2. Let $X \in \mathcal{C H}_{h, p, q}$ and $Y \in \mathcal{C H}_{h, p+1, q^{\prime}}$. Then by Proposition 2.2

$$
\begin{aligned}
T I(X)= & \left(\varphi_{22}-2 \varphi_{23}+\varphi_{33}\right)(q+3 p)+\left(4 \varphi_{23}+\varphi_{33}\right) h \\
& +\left(6 \varphi_{22}-4 \varphi_{23}-\varphi_{33}\right) \\
T I(Y)= & \left(\varphi_{22}-2 \varphi_{23}+\varphi_{33}\right)\left(q^{\prime}+3(p+1)\right)+\left(4 \varphi_{23}+\varphi_{33}\right) h \\
& +\left(6 \varphi_{22}-4 \varphi_{23}-\varphi_{33}\right) .
\end{aligned}
$$

Hence

$$
T I(X)-T I(Y)=\left(\varphi_{22}-2 \varphi_{23}+\varphi_{33}\right)\left(q-q^{\prime}-3\right)<0
$$

if and only if $q^{\prime}>q-3$.

3. It follows from relation (6).

Dually we have the following result.

Rev.Mate.Teor.Aplic. (ISSN print: 1409-2433; online: 2215-3373) Vol. 23(1): 277-289, January 2016 
Theorem 2.7 Let TI be a VDB topological index induced by the numbers $\left\{\varphi_{i j}\right\}$. Let $h \geq 3$ and $0 \leq p \leq\left\lfloor\frac{1}{2}(h-2)\right\rfloor$. Assume that $\varphi_{22}-2 \varphi_{23}+\varphi_{33}<0$. Then

1. $T I\left(\mathcal{C H}_{h, p, i}\right)>T I\left(\mathcal{C H}_{h, p, i+1}\right)$ for all $i=0, \ldots, h-2 p-3$.

2. $T I\left(\mathcal{C H}_{h, p, q}\right)>T I\left(\mathcal{C H}_{h, p+1, q^{\prime}}\right)$ if and only if $q^{\prime}>q-3$.

3. $T I\left(\mathcal{C H}_{h, p, q}\right)=T I\left(\mathcal{C H}_{h, p+1, q^{\prime}}\right)$ if and only if $q^{\prime}=q-3$.

Proof. The proof is similar to the proof of Theorem 2.6

Theorems 2.6 and 2.7 can be nicely illustrated organizing the information in a table. First it is important to notice that a consequence of Lemmas 2.4 and 2.5 is the fact that it is possible to partition the set $\mathcal{C H}_{h}$ of all catacondensed hexagonal systems as a disjoint union

$$
\mathcal{C H}_{h}=\bigcup \mathcal{C H} \mathcal{H}_{h, p, q}
$$

where $(p, q)$ runs through the set

$$
\left\{(p, q) \in \mathbb{N} \times \mathbb{N}: 0 \leq p \leq\left\lfloor\frac{1}{2}(h-2)\right\rfloor \text { and } 0 \leq q \leq h-2(p+1)\right\} .
$$

Example 2.8 We first consider an example when $h$ is even. Assume that $h=8$. Then by Lemmas 2.4 and 2.5. $0 \leq p \leq 3$ and $0 \leq q \leq 8-2(p+1)$. The partition of $\mathrm{CH}_{8}$ is displayed in the following table:

\begin{tabular}{|l|l|l|l|l|l|l|l|l|l|}
\hline $\mathcal{C H}_{8,0,0}$ & $\mathcal{C H}_{8,0,1}$ & $\mathcal{C H}_{8,0,2}$ & $\mathcal{C H}_{8,0,3}$ & $\mathcal{C H}_{8,0,4}$ & $\mathcal{C H}_{8,0,5}$ & $\mathcal{C H}_{8,0,6}$ & & & \\
\hline & & & $\mathcal{C H}_{8,1,0}$ & $\mathcal{C H}_{8,1,1}$ & $\mathcal{C H}_{8,1,2}$ & $\mathcal{C H}_{8,1,3}$ & $\mathcal{C H}_{8,1,4}$ & & \\
\hline & & & & & $\mathcal{C H}_{8,2,0}$ & $\mathcal{C H}_{8,2,1}$ & $\mathcal{C H}_{8,2,2}$ & \\
\hline & & & & & & & & & $\mathcal{C H}_{8,3,0}$ \\
\hline
\end{tabular}

Theorem 2.6] should be read as follows: if $\varphi_{22}-2 \varphi_{23}+\varphi_{33}>0$ then moving to the right in any row the value of the topological index TI strictly increases. Moving in the same column the value of TI is constant. Consequently,

1. The minimal value of $T$ I over $\mathrm{CH}_{8}$ is attained in the linear chain $L_{8}$ since $L_{8} \in \mathcal{C H}_{8,0,0}$. The maximal value of $T I$ over $\mathcal{C H}_{8}$ is attained in the system $E_{8}$ since $E_{8} \in \mathcal{C H}_{8,3,0}$.

2. More generally, there are exactly 10 different values of TI over $\mathrm{CH}_{8}$, being the $i^{\text {th }}$-maximal value of TI any hexagonal system which belongs to the $i^{\text {th }}$ column of the table above. 
3. For each $0 \leq p \leq 3$, the minimal value of $T I$ over the set

$$
\bigcup_{0 \leq q \leq 8-2(p+1)} \mathcal{C H}_{8, p, q}
$$

is attained in a system belonging to $\mathcal{C H}_{8, p, 0}$ and the maximal value in a system belonging to $\mathcal{C H}_{8, p, 8-2(p+1)}$. In particular, when $p=0$ then

$$
\bigcup_{0 \leq q \leq 8-2(p+1)} \mathcal{C} \mathcal{H}_{8, p, q}=\bigcup_{0 \leq q \leq 6} \mathcal{C} \mathcal{H}_{8,0, q}
$$

is the set of hexagonal chains with 8 hexagons. So among all hexagonal chains with 8 hexagons, the minimal value of $T I$ is attained in $L_{8} \in \mathcal{C H}_{8,0,0}$ and the maximal value in the zig-zag chain $Z_{8} \in \mathcal{C H}_{8,0,6}$.

4. Two hexagonal systems $U \in \mathcal{C H}_{8, p, q}$ and $V \in \mathcal{C H}_{8, p^{\prime}, q^{\prime}}$ in $\mathcal{C H}_{8}$ have equal $T I$ if and only if $p-p^{\prime}=3\left(q-q^{\prime}\right)$.

Example 2.9 Assume that $h=9$. Then by Lemmas 2.4 and 2.5 . $0 \leq p \leq 3$ and $0 \leq q \leq 7-2 p$. The partition of $\mathcal{C H}_{9}$ is displayed in the following table:

\begin{tabular}{|l|l|l|l|l|l|l|l|l|l|l|}
\hline $\mathcal{C H}_{9,0,0}$ & $\mathcal{C H}_{9,0,1}$ & $\mathcal{C H}_{9,0,2}$ & $\mathcal{C H}_{9,0,3}$ & $\mathcal{C H}_{9,0,4}$ & $\mathcal{C H}_{9,0,5}$ & $\mathcal{C H}_{9,0,6}$ & $\mathcal{C H}_{9,0,7}$ & & & \\
\hline & & & $\mathcal{C H}_{9,1,0}$ & $\mathcal{C H}_{9,1,1}$ & $\mathcal{C H}_{9,1,2}$ & $\mathcal{C H}_{9,1,3}$ & $\mathcal{C H}_{9,1,4}$ & $\mathcal{C H}_{9,1,5}$ & & \\
\hline & & & & & & $\mathcal{C H}_{9,2,0}$ & $\mathcal{C H}_{9,2,1}$ & $\mathcal{C H}_{9,2,2}$ & $\mathcal{C H}_{9,2,3}$ & \\
\hline & & & & & & & & $\mathcal{C H}_{9,3,0}$ & $\mathcal{C H}_{9,3,1}$ \\
\hline
\end{tabular}

Theorem 2.7 should be read as follows: if $\varphi_{22}-2 \varphi_{23}+\varphi_{33}<0$ then moving to the right in any row the value of the topological index TI strictly decreases. Moving in the same column the value of TI is constant. Consequently, a dual statement to the one given in Example 2.8 holds.

In conclusion, for every $h \geq 3$ the information of a VDB topological index TI over $\mathcal{C H}_{h}$ is completely determined from a table constructed as in Examples 2.8 and 2.9. based on the partition (7) and Theorems 2.6 and 2.7. 
Corollary 2.10 Let TI be a VDB topological index induced by the numbers $\left\{\varphi_{i j}\right\}$. If $\varphi_{22}-2 \varphi_{23}+\varphi_{33}>0$ (resp. $\left.\varphi_{22}-2 \varphi_{23}+\varphi_{33}<0\right)$ then

1. The minimal value of $T I$ over $\mathcal{C H}_{h}$ is attained in $L_{h}$ (resp. $E_{h}$ ) and the maximal value in $E_{h}\left(\right.$ resp. $\left.L_{h}\right)([17])$.

2. There are exactly $h-1+\left\lfloor\frac{1}{2}(h-2)\right\rfloor$ different values of TI over $\mathcal{C H}_{h}$. The $(i)^{\text {th }}$-maximal (resp. $(i)^{\text {th }}$-minimal) value of $T I$ over $\mathcal{C H}_{h}$ is attained in a hexagonal system belonging to

$$
\begin{array}{cc}
\mathcal{C H}_{h, 0, i-1} & \text { if } 1 \leq i \leq h-1 \\
\mathcal{C} \mathcal{H}_{h, i-h+1,3 h-2 i-4} & \text { if } h \leq i \leq h-1+\left\lfloor\frac{1}{2}(h-2)\right\rfloor .
\end{array}
$$

3. For each $0 \leq p \leq\left\lfloor\frac{1}{2}(h-2)\right\rfloor$, the minimal (resp. maximal) value of TI over the set

$$
\bigcup_{0 \leq q \leq h-2(p+1)} \mathcal{C} \mathcal{H}_{h, p, q}
$$

is attained in a system belonging to $\mathcal{C H}_{h, p, 0}$ and the maximal (resp. minimal) value in a system belonging to $\mathcal{C H}_{h, p, h-2(p+1)}$. In particular, when $p=0$ then

$$
\bigcup_{0 \leq q \leq h-2(p+1)} \mathcal{C H}_{h, p, q}=\bigcup_{0 \leq q \leq h-2} \mathcal{C H}_{h, 0, q}
$$

is the set of hexagonal chains with h hexagons. So among all hexagonal chains with h hexagons, the minimal (resp. maximal) value of TI is attained in $L_{h} \in \mathcal{C H}_{h, 0,0}$ and the maximal (resp. minimal) value in the zig-zag chain $Z_{h} \in \mathcal{C H}_{h, 0, h-2}$.

4. Two hexagonal systems $U \in \mathcal{C H}_{h, p, q}$ and $V \in \mathcal{C H}_{h, p^{\prime}, q^{\prime}}$ in $\mathcal{C H}_{h}$ have equal $T I$ if and only if $p-p^{\prime}=3\left(q-q^{\prime}\right)$.

Note that in order to apply Corollary 2.10 to a specific VDB topological index $T I$ induced by the numbers $\left\{\varphi_{i j}\right\}$, we first must determine the sign of $\varphi=\varphi_{22}-2 \varphi_{23}+\varphi_{33}$. Clearly by Proposition 2.2 if $\varphi=0$ then $T I$ is constant over $\mathcal{C H}_{h}$. In the following table we compute the values of $\varphi$ for the main VDB topological indices:

\begin{tabular}{|c|c|c|c|c|c|c|c|c|}
\hline & $\mathcal{F Z}$ & $\mathcal{S Z}$ & $\chi$ & $\mathcal{H}$ & $\mathcal{G A}$ & $\mathcal{S C}$ & $\mathcal{A B C}$ & $\mathcal{A Z}$ \\
\hline$\varphi$ & 0 & 1 & .0168 & .0333 & .0404 & .0138 & -.0404 & 3.3906 \\
\hline
\end{tabular}




\section{References}

[1] Berrocal, L.; Olivieri, A.; Rada, J. (2014) "Extremal values of vertexdegree-based topological indices over hexagonal systems with fixed number of vertices", Appl. Math. Comp. 243: 176-183.

[2] Cruz, R.; Giraldo, H.; Rada, J. (2013) "Extremal values of vertex-degree topological indices over hexagonal systems", MATCH Commun. Math. Comput. Chem. 70: 501-512.

[3] Devillers, J; Balaban, A.T., Eds. (1999) Topological Indices and Related Descriptors in QSAR and QSPR. Gordon \& Breach, Amsterdam.

[4] Estrada E.; Torres L.; Rodríguez L.; Gutman, I. (1998) “An atom-bond connectivity index: Modelling the enthalpy of formation of alkanes", Indian J. Chem. 37A: 849-855.

[5] Furtula B.; Graovac A.; Vukičević D. (2010) "Augmented Zagreb index”, J. Math. Chem. 48(2): 370-380.

[6] Furtula B.; Gutman, I.; Dehmer M. (2013) "On structure-sensitivity of degree-based topological indices", Appl. Math. Comput. 219: 8973-8978.

[7] Gutman, I.; Trinajstić, N. (1972) "Graph theory and molecular orbitals. Total $\phi$-electron energy of alternant hydrocarbons", Chem. Phys. Lett. 17(4): $535-538$.

[8] Gutman, I. (1985) "Topological properties of benzenoid systems. XLI. Carbon-carbon bond types and connectivity indices of benzenoid hydrocarbons", J. Serb. Chem. Soc. 50(9-10): 451-455.

[9] Gutman, I.; Cyvin, S.J. (1989) Introduction to the Theory of Benzenoid Hydrocarbons. Springer-Verlag, Berlin.

[10] Gutman, I. (2013) "Degree-based topological indices", Croat. Chem. Acta 86(4): 351-361.

[11] Gutman, I.; Tošović, J. (2013) "Testing the quality of molecular structure descriptors. Vertex-degree-based topological indices", J. Serb. Chem. Soc. 78(6): 805-810.

[12] Kier, L.B.; Hall, L.H. (1976) Molecular Connectivity in Chemistry and Drug Research. Academic Press, New York. 
[13] Kier, L.B.; Hall, L.H. (1986) Molecular Connectivity in Structure-Activity Analysis. Wiley, New York.

[14] Li, X.; Shi, Y. (2008) "A survey on the Randić index”, MATCH Commun. Math. Comput. Chem. 59: 127-156.

[15] Li, X.; Shi, Y.; Wang, L. (2008) "An updated survey on the Randić index", in: I. Gutman \& B. Furtula (Eds.) Recent Results in the Theory of Randić Index, Mathematical Chemistry Monograph, Vol. 6, Univ. Kragujevac, Kragujevac: 9-47.

[16] Rada, J. (2002) "Bounds for the Randić index of catacondensed systems", Util. Math. 62: 155-162.

[17] Rada, J.; Cruz, R.; Gutman, I. (2013) "Vertex-degree-based topological indices of catacondensed hexagonal systems", Chem. Phys. Lett. 572: 154157.

[18] Rada, J.; Cruz, R. (2014) "Vertex-degree-based topological indices over graphs", MATCH Commun. Math. Comput. Chem. 72(3): 603-616.

[19] Rada, J.; Cruz, R.; Gutman, I. (2014) "Benzenoid systems with extremal vertex-degree-based topological indices", MATCH Commun. Math. Comput. Chem. 72(1): 125-136.

[20] Randić, M. (1975) "Characterization of molecular branching", J. Am. Chem. Soc. 97(23): 6609-6615.

[21] Todeschini, R.; Consonni, V. (2000) Handbook of Molecular Descriptors. Wiley-VCH, Weinheim.

[22] Vukičević, D.; Furtula, B. (2009) "Topological index based on the ratios of geometrical and arithmetical means of end-vertex degrees of edges", $J$. Math. Chem. 46(4): 1369-1376.

[23] Zhong, L. (2012) “The harmonic index for graphs”, Appl. Math. Lett. 25(3): 561-566.

[24] Zhou, B.; Trinajstić, N. (2009) "On a novel connectivity index", J. Math. Chem. 46(4): 1252-1270. 
